\title{
Expression Levels of Some Antioxidant and Epidermal Growth Factor Receptor Genes in Patients with Early-Stage Non-Small Cell Lung Cancer
}

\author{
Giuseppe De Palma, ${ }^{1}$ Paola Mozzoni, ${ }^{2,3}$ Olga Acampa, ${ }^{2,3}$ Eveline Internullo, ${ }^{4}$ \\ Paolo Carbognani, ${ }^{4}$ Michele Rusca, ${ }^{4}$ Matteo Goldoni, ${ }^{2,3}$ Massimo Corradi, ${ }^{3}$ \\ Marcello Tiseo, ${ }^{5}$ Pietro Apostoli, ${ }^{1}$ and Antonio Mutti ${ }^{3}$ \\ ${ }^{1}$ Department of Experimental and Applied Medicine, Section of Occupational Health and Industrial Hygiene, University of Brescia, \\ Piazzale Spedali Civili, 1, 25123 Brescia, Italy \\ ${ }^{2}$ ISPESL Research Center at the University of Parma, via Gramsci 14, 43100 Parma, Italy \\ ${ }^{3}$ Laboratory of Industrial Toxicology, Department of Clinical Medicine Nephrology and Health Sciences, University of Parma, \\ via Gramsci 14, 43100 Parma, Italy \\ ${ }^{4}$ Thoracic Surgery Unit, University of Parma, via Gramsci 14, 43100 Parma, Italy \\ ${ }^{5}$ Division of Medical Oncology, University of Parma, via Gramsci 14, 43100 Parma, Italy
}

Correspondence should be addressed to Giuseppe De Palma, depalma@med.unibs.it

Received 24 November 2009; Revised 21 January 2010; Accepted 27 January 2010

Academic Editor: Hiroshi Sugiyama

Copyright (C) 2010 Giuseppe De Palma et al. This is an open access article distributed under the Creative Commons Attribution License, which permits unrestricted use, distribution, and reproduction in any medium, provided the original work is properly cited.

\begin{abstract}
This study was aimed at: (i) investigating the expression profiles of some antioxidant and epidermal growth factor receptor genes in cancerous and unaffected tissues of patients undergoing lung resection for non-small cell lung cancer (NSCLC) (cross-sectional phase), (ii) evaluating if gene expression levels at the time of surgery may be associated to patients' survival (prospective phase). Antioxidant genes included heme oxygenase 1 (HO-1), superoxide dismutase-1 (SOD-1), and -2 (SOD-2), whereas epidermal growth factor receptor genes consisted of epidermal growth factor receptor (EGFR) and v-erb-b2 erythroblastic leukaemia viral oncogene homolog 2 (HER-2). Twenty-eight couples of lung biopsies were obtained and gene transcripts were quantified by Real Time RT-PCR. The average follow-up of patients lasted about 60 months. In the cancerous tissues, antioxidant genes were significantly hypo-expressed than in unaffected tissues. The HER-2 transcript levels prevailed in adenocarcinomas, whereas EGFR in squamocellular carcinomas. Patients overexpressing HER-2 in the cancerous tissues showed significantly lower 5-year survival than the others.
\end{abstract}

\section{Introduction}

Lung cancer, in particular non-small cell lung cancer (NSCLC), is the leading cause of cancer related deaths worldwide, given its incidence and poor prognosis mainly due to delay in diagnosis [1]. Molecular heterogeneity in the genomics and/or proteomics of NSCLC may underlie a different clinical outcome and response to therapy of patients with similar clinical stage and histopathology. Among molecular markers, expression profiles of antioxidant genes and proto-oncogenes, identified by microarrays or quantitative reverse transcriptase PCR (qRT-PCR), may significantly affect the clinical outcome of NSCLC [2-4].
Oxidative stress plays a key role in both development and progression of NSCLC. Reactive oxygen species (ROS) can induce DNA damage that, if unrepaired, may lead to disruption of gene transcription as well as to interference with DNA methylation [5, 6]. On the other hand, ROS may up-regulate transcription factors as MAP-kinase/AP-1 and NF-kB that, in turn, induce the expression of several genes involved in key cellular pathways [7, 8]. Among these, there is heme oxygenase-1 (HO-1), a microsomal heat shock protein that catalyzes the first and rate-limiting step in heme catabolism and shows high inducibility by several stimuli [9]. Expressed in airway epithelial cells and alveolar macrophages, it displays both a cytoprotective 
and antioxidant role [10]. Cytosolic and mitochondrial superoxide dismutases (SOD-1 and SOD-2, resp.) play also a prominent role in the pulmonary antioxidant defense system [11], catalyzing the dismutation of superoxide anion to hydrogen peroxide and water. Experimental evidence is available showing that ROS may induce the ligand independent phosphorylation of the Epidermal Growth Factor Receptor (EGFR) through oxidized src kinases [12, 13]. Moreover, the expression of the receptor is induced in conditions of oxidative stress [14]. The EGFR belongs to the ErbB family of transmembrane receptors including at least four isoforms, namely erbB-1 (EGFR), erbB-2 (HER-2), erbB-3 (HER3), and erbB-4 (HER4), whose role in lung carcinogenesis as oncogenes is generally accepted [15]. Following the binding of epidermal growth factor (EGF)-like ligands, these receptors homo/hetero-dimerize on the cell surface and activate the cytosolic tyrosine-kinase domain, with downstream stimulation of intracellular signalling pathways, which are essential for cancer development and progression [16, 17]. Both EGFR and HER-2, having an 85\% homology in amino acid residues, trigger similar down stream signal event. There are no known ligands for HER-2 that is the preferred heterodimerization partner of this family of receptors [16].

The main aim of the present study was to evaluate differences in the expression profiles of antioxidant ( $\mathrm{HO}-1$, $S O D-1, S O D-2)$ and epidermal growth factor genes (EGFR, $H E R-2)$ in both cancerous and unaffected lung tissues of clinical early-stage NSCLC patients.

As a further aim, we evaluated the influence of gene expression levels at the time of surgery on further patients' survival.

\section{Materials and Methods}

2.1. Study Design. This was an observational study on consecutive patients with a single lung lesion either proven or suspected to be an early clinical stage (I or II) of NSCLC and undergoing lung resection with curative intent. The study was both cross-sectional and prospective in nature. In the cross-sectional phase, we evaluated the gene expression levels in both cancerous and unaffected lung tissues, whereas in the prospective phase, we evaluated the influence, if any, of gene expression levels at the time of surgery on the patients' survival times.

Patients' enrollment proceeded from June to August 2002 and from April 2003 to April 2004, with the interruption being due to a temporary lack of adequate systems of biopsy storage. Exclusion criteria were clinical T3 tumors and a history of malignancy in the previous 5 years. Informed consent was obtained from each patient at the time of enrollment, and sample collection and storage were performed according to the principles laid down in the Declaration of Helsinki (1964). The study received approval by the local Ethics Committee.

Preoperative work-up included medical history and examination, oncological staging and functional evaluation. All patients were staged by chest and abdomen CT scans; in addition, brain CT scan, abdominal ultrasound, or bone scintigraphy were performed if indicated. Any death occurring within 30 days from surgery or during the same hospitalization was defined as postoperative mortality and cases were excluded from survival analysis.

Survival was calculated from the day of surgery to death or last follow-up (1 April 2008), provided that patients did not receive any palliative therapy.

2.2. Sample Collection and Storage. Small pieces (about 0.5$1 \mathrm{~cm}^{3}$ ) of lung biopsies, from both cancerous and unaffected tissues of the resected specimen, were collected into tubes containing the RNAlater reagent (Ambion Inc., Austin, TX, USA) and stored at $-80^{\circ} \mathrm{C}$ until analysis. Unaffected tissue samples underwent intraoperative frozen section examination to exclude tumor infiltration.

2.3. RNA Isolation and cDNA Synthesis. Total RNA was isolated by the TRIzol reagent (Invitrogen, Carlsbad, CA, USA) and digested with DNase I (DNA-free, Ambion Inc., Austin, TX, USA). RNA quality was checked by $1 \%$ TBE agarose gel electrophoresis, using a denaturing loading buffer (RNA Ladder, New England Biolabs Inc., Beverly, MA, USA). Total RNA quantification was carried out by the RiboGreen probe (Molecular Probes, Eugene, OR, USA) on a Cary Eclipse fluorescence spectrophotometer equipped with a microplate reader (Varian Inc, Scientific Instruments, Palo Alto, CA, USA). cDNA was synthesized using 250-500 ng of total RNA, 250 ng of random exhamer primers (Invitrogen, Carlsbad, CA, USA), and $200 \mathrm{U}$ of SuperScript II Reverse Transcriptase (Invitrogen, Carlsbad, CA, USA), following the manufacturer's recommended experimental conditions.

2.4. Gene Expression. HO-1, SOD-1, SOD-2, EGFR, and HER-2 gene expression was assessed by Real-Time qRTPCR on an iCycler iQ Multicolor RealTime PCR Detection System (Bio-Rad, Hercules, CA, USA). The PCR mixture contained $2 \mu \mathrm{L}$ of template cDNA, $400 \mathrm{nM}$ of primers, 100 nM of FAM-conjugated Universal Probe (Roche, Diagnostic Mannheim, Germany), and $2 \times$ QuantiTect Probe RT-PCR Master Mix (Qiagen, Hilden, Germany) and water to $25 \mu \mathrm{L}$. Table 1 presents the specific primers, spanning the exonexon junctions, and probes, designed by the ProbeFinder software (Roche Diagnostics, Mannheim, Germany). The amplification protocol consisted of 15 minutes at $95^{\circ} \mathrm{C}$ followed by 40 cycles at $94^{\circ} \mathrm{C}$ for $20 \mathrm{~s}$ and at $60^{\circ} \mathrm{C}$ for 1 minute. Duplicate assays were run for each sample and each plate included a standard curve and a negative control.

The relative transcript quantification was calculated by the geNorm_algorithm for Microsoft Excel [18] after normalization with $\beta_{2}$-microglobulin $\left(\beta_{2} M\right)$, phosphoglycerate kinase 1 (PGK1), succinate dehydrogenase complex subunit A (SDHA), hypoxanthine-guanine phosphoribosyltransferase (HPRT), and ribosomal protein L13 (RPL13) genes, and expressed in terms of arbitrary units (a.u.).

2.5. Statistical Analysis. Statistical analysis was performed by the SPSS 15.0 for Windows software. Variables were not normally distributed, also after logarithmic transformation, 
TABLE 1: Nucleotide sequences for primers and probes used in the present study.

\begin{tabular}{|c|c|c|c|}
\hline Genes & Forward primers & Reverse primers & Probes \\
\hline$S O D-1$ & 5'TCATCAATTTCGAGCAGAAGG3' & 5 CAGGCCTTCAGTCAGTCCTTT3' & 5'FAM TGGGGAAG3'DQ \\
\hline$S O D-2$ & 5'TGGTGGAGAACCCAAAGG3' & 5'GTCAAAGGAACCAAAGTCACG3' & 5'FAM GCTGGAAG3'DQ \\
\hline $\mathrm{HO}-1$ & 5'GGGTGATAGAAGAGGCCAAGA3' & 5'AGCTCCTGCAACTCCTCAAA3' & 5'FAM CATCCAGC3'DQ \\
\hline EGFR & 5'ACACAGAATCTATACCCACCAGAGT3' & 5'ATCAACTCCCAAACGGTCAC3' & 5'FAM TCTGGAGC3'DQ \\
\hline HER-2 & 5'GATCCGGAAGTACACGATGC3' & 5'GCTCCGCTAGGTGTCAGC3' & 5'FAM TGGTGGAG3'DQ \\
\hline$P G K 1$ & 5'GGAGAACCTCCGCTTTCAT3' & 5'CTGGCTCGGCTTTAACCTT3' & 5'FAM GGAGGAAG3'DQ \\
\hline$B 2 M$ & 5'TTCTGGCCTGGAGGCTATC3' & 5'TCAGGAAATTTGACTTTCCATTC3' & 5'FAM CATCCAGC3'DQ \\
\hline SDHA & 5'AGAAGCCCTTTGAGGAGCA3' & 5'CGATTACGGGTCTATATTCCAGA3' & 5'FAM GGAGGAAG3'DQ \\
\hline$H P R T$ & 5'TGACCTTGATTTATTTTGCATACC3' & 5'CGAGCAAGACGTTCAGTCCT3' & 5'FAM GCTGAGGA3'DQ \\
\hline RPL13 & 5'ACAGCTGCTCAGCTTCACCT3' & 5'TGGCAGCATGCCATAAATAG3' & 5'FAM CAGTGGCA3'DQ \\
\hline
\end{tabular}

Legend: $\mathrm{FAM}=$ fluorescein; $\mathrm{DQ}=$ dark quencher dye.

hence data were analyzed by nonparametric tests. Comparisons between two independent or two related samples were performed by the Mann-Whitney U or the Wilcoxon's test. The ratios of gene expression levels in the cancerous versus the unaffected tissues were recoded into dichotomic variables (values up to or higher than one) that were used to evaluate survival curves by the Kaplan Meyer analysis with log-rank (Mantel-Cox) test. The Cox regression analysis was used to study the effect of multiple covariates on survival curves. Two patients deceased for causes surely unrelated to NSCLC (a postoperative death and pulmonary embolism after discharge) were excluded from analyses of survival.

\section{Results and Discussion}

During the study period, 89 patients were admitted at a single center as affected by a lung lesion proven or suspected to be an NSCLC at clinical stage I or II. Of these: 10 were excluded for clinical stage $\mathrm{T}_{3} \mathrm{~N}_{0} \mathrm{M}_{0}$ and 15 for a history of malignancy in the previous 5 years. Nine patients did not give consent to the study, thus 55 were enrolled. In eight cases, the lung lesion was too small $(1.5 \mathrm{~cm}$ maximum diameter) or completely endobronchial, so that the surgeon did not perform any biopsy in order not to spoil the final pathology exam. Of the 47 patients undergoing biopsy, 19 were weeded out because frozen section on the nodule revealed benign lesions $(n=7)$ or metastases from another primary site $(n=7)$ or carcinoid tumors $(n=5)$. Therefore, 28 couples of biopsies (cancerous and unaffected tissues) constitute the final study sample. There were 5 females and 23 males, mean aged 70 years (range 55-82). Twenty-six out of 28 were either previous or current smokers, with a mean tobacco exposure of 50.5 pack/years. Twenty-four lobectomies, three pneumonectomies, and one anatomical segmentectomy were performed, associated with standard lymphadenectomy. There were 14 adenocarcinomas (ADC), 13 squamous cell carcinomas (SCC), and 1 undifferentiated carcinoma. Despite all patients were clinically stage I or II at preoperative examinations, final pathology [19] revealed the following: stage I in 20 cases ( 6 stage Ia and 14 stage Ib), stage II in 5 ( 1 IIa and 4 IIb), and stage IIIa in 3 . None of
Table 2: Distribution of gene transcript levels (medians and interquartile (25th-75th) ranges of values in arbitrary units, a.u) in cancerous and unaffected tissues. Results of paired sample analysis (Wilcoxon's test) are shown $(P)$.

\begin{tabular}{lccc}
\hline $\begin{array}{l}\text { Gene } \\
\text { transcripts }\end{array}$ & $\begin{array}{c}\text { Cancerous tissue, } \\
\text { a.u. }\end{array}$ & $\begin{array}{c}\text { Unaffected tissue, } \\
\text { a.u. }\end{array}$ & $P$ \\
\hline HO-1 & $0.10(0.07-0.15)$ & $0.16(0.13-0.22)$ & .031 \\
SOD-1 & $0.13(0.09-0.15)$ & $0.18(0.14-0.19)$ & .006 \\
SOD-2 & $0.17(0.09-0.23)$ & $0.26(0.18-0.36)$ & .001 \\
EGFR & $0.07(0.04-0.09)$ & $0.05(0.03-0.07)$ & .258 \\
HER-2 & $0.23(0.09-0.37)$ & $0.23(0.16-0.35)$ & .228 \\
\hline
\end{tabular}

the patients received adjuvant therapy or any patient was lost at follow-up. At the time of data analysis (70 months after the first patient was enrolled), 14 (50\%) of the patients had died: 12 by disease recurrence and two by tumor unrelated causes, by postoperative death and pulmonary embolism after discharge, respectively. The overall 5-year survival was $50 \%$ and it became $56 \%$ if considering only stage I and II patients. Median follow-up for the 14 patients alive was 58.9 months (range 48-70).

Table 2 shows the average levels of investigated transcripts both in cancerous and unaffected lung tissues. In two cases, we failed to characterize the transcript levels, because of poor quality RNA.

The HO-1,SOD-1, and SOD-2 transcript levels were significantly lower in the cancerous tissue, whereas no significant difference was apparent for both EGFR and HER-2 mRNA levels. After stratification of patients by histopathology ( 25 subjects, excluding the only patient with diagnosis on undifferentiated carcinoma), some difference was apparent only for EGFR and HER-2 transcripts. The levels of HER-2 mRNA were significantly higher in ADC than SCC tumor samples (median values of 0.38 (interquartile range 0.24 $0.51)$ a.u. versus $0.10(0.07-0.13)$ a.u., $P<.001)$, whereas the difference was not significant for the EGFR transcript (Figure 1(a)). Figure 1(b) shows the ratios of EGFR and HER-2 transcript levels in the cancerous versus unaffected tissues. EGFR levels prevailed significantly in subjects with 


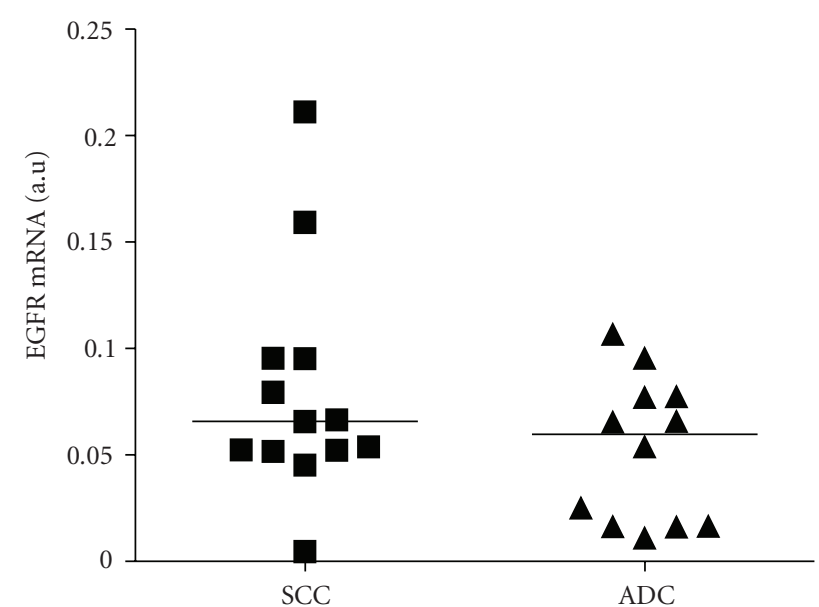

(a)

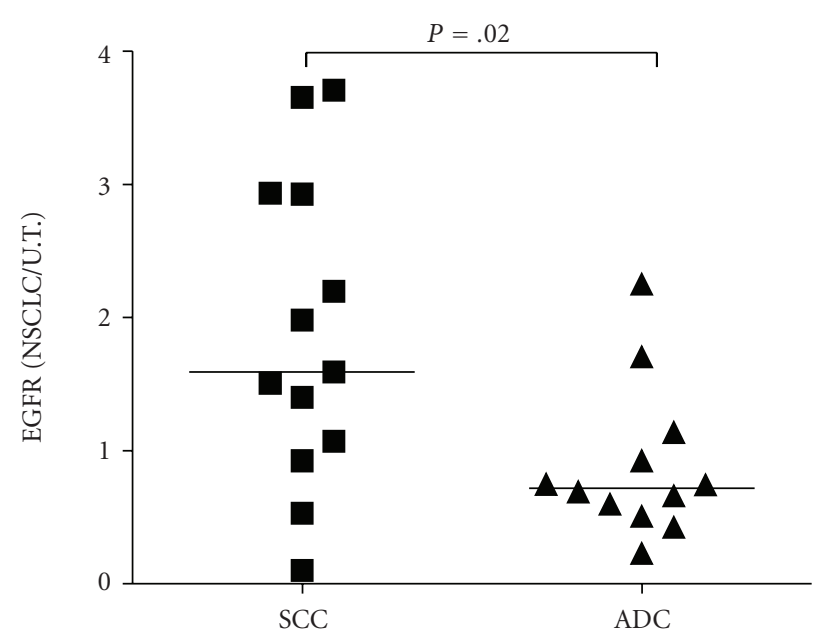

(c)

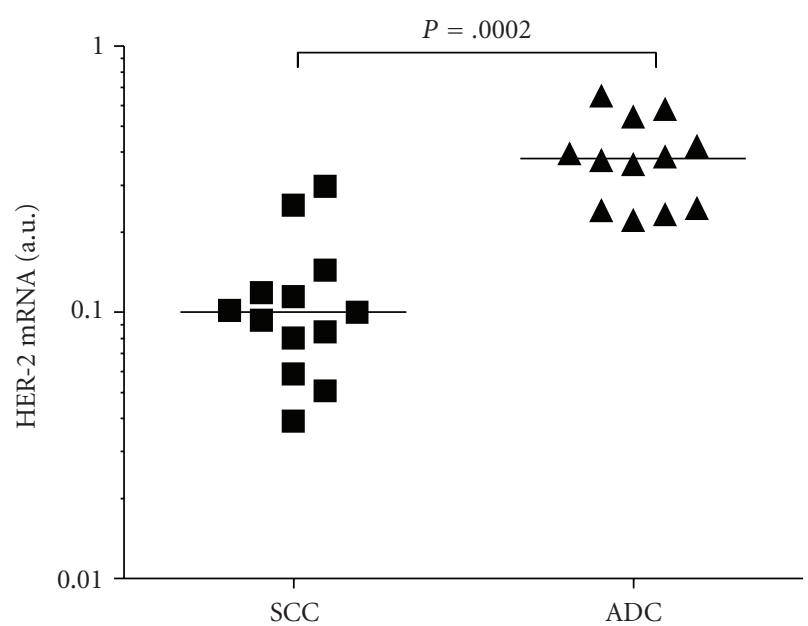

(b)

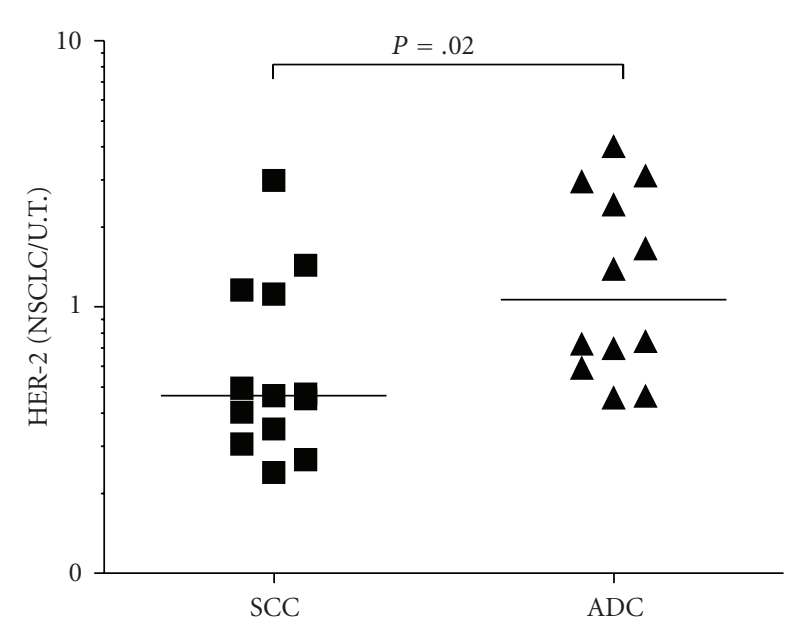

(d)

FIGURE 1: (a) EGFR and HER-2 transcript levels (as arbitrary units, a.u.) in squamocellular carcinoma (SCC) and adenocarcinoma (ADC) samples. Lines represent medians of values. (b) Expression ratio levels of the same genes in the cancerous (C.T.) versus unaffected tissues (U.T.). Lines represent medians of values.

SCC as compared to those with ADC (median values of 1.59 (interquartile range 1.00-2.93) a.u. versus $0.72(0.53-1.08)$ a.u., $P=.019$ ), whereas the opposite held true for HER-2 (median values of 1.06 (interquartile range $0.62-2.82$ ) a.u. versus $0.46(0.33-1.14)$ a.u., $P=.019)$.

Then, we proceeded to analyze the survival times on 24 patients (in two cases, gene expression results were unavailable and two cases were excluded, given their death by cancer unrelated causes). Table 3 shows that subjects overexpressing HER-2 in the cancerous tissue as compared to the unaffected one displayed survival times about three times lower, on average, than the rest of subjects. In agreement with this, 5-year survivors showed significantly lower HER-2 transcript levels in the cancerous versus the unaffected tissue than patients dead within 5 years from surgery (medians (interquartile ranges) of $0.48(0.32-0.73)$ a.u. versus 1.39 (0.70-2.95) a.u., $P<.05)$.

The effect of the HER-2 transcript ratio on survival was confirmed by the Kaplan Meier analysis (Figure 2). A Cox regression model including also age, sex, tumor stage, and histology as covariates confirmed the role of HER-2 transcript ratio as main factor $(P=.012)$ affecting the patients' survival, whereas other covariates showed no effect. In the same model, the strength of association between longer survival times and HER-2 transcript ratio values higher than 1 was 11.42 (95\% confidence interval: $1.74-74.84$ ).

The present study shows that the expression levels of genes displaying antioxidant and cytoprotective properties, such as SOD-1, SOD-2, and HO-1, are depressed in lung cancer as compared to the unaffected lung tissue. These results indirectly confirm the association of oxidative stress with the neoplastic phenotype. Superoxide dismutases are generally regarded as first line antioxidant enzymes, given their unique role in detoxification of superoxide radicals. Previous studies investigating on SOD expression in human lung cancer produced variable results (reviewed in [20]). With the exception of one study, finding raised $S O D-2$ (and not SOD-1) transcript levels (by Northern blot analysis) in 


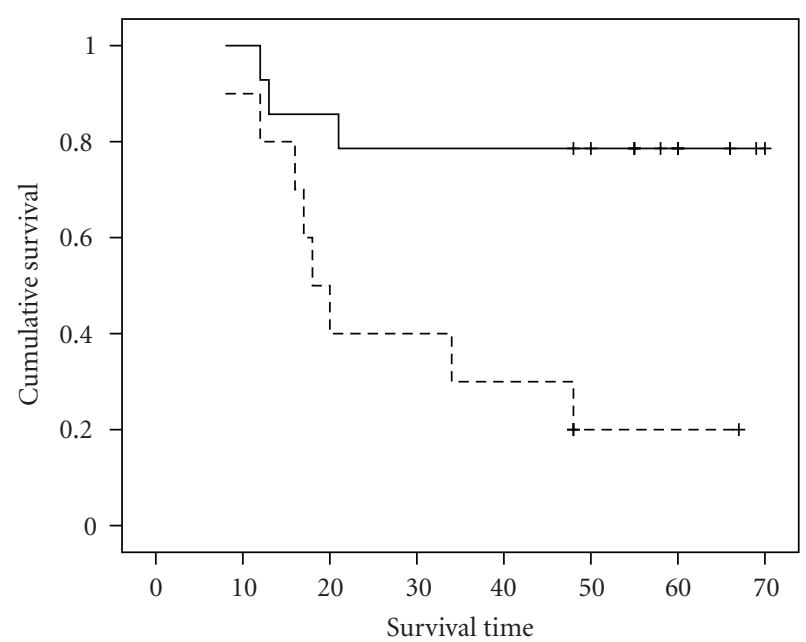

FIgURE 2: Kaplan-Meier plot of survival times in NSCLC patients classified by expression ratio levels of HER-2 transcript in cancerous versus unaffected tissues (up to or higher than one). The dashed line represents subjects over-expressing HER-2 mRNA in the cancerous versus unaffected tissues, whereas the continuous line indicates subjects with lower expression levels of HER-2 mRNA in cancerous versus unaffected tissue samples. Crosses indicate censored subjects, represented by patients still alive at 1st April 2008.

TABle 3: Average survival times in subjects classified according to the expression levels of different transcript in cancerous (C.T.) versus unaffected (U.T.) tissues. Medians and interquartile (25th75th) ranges of values are shown, as well as the results of the MannWhitney U test $(P)$.

\begin{tabular}{lccc}
\hline \multirow{2}{*}{ Transcripts } & \multicolumn{2}{c}{ Survival (months) } & \multirow{2}{*}{ C. T. / U.T $>1$} \\
& $48.00(33.00-63.00)$ & $48.00(16.50-59.00)$ & .659 \\
HO-1 & $52.50(11.00-60.00)$ & $48.00(17.50-63.00)$ & .865 \\
SOD-1 & $18.00(14.50-57.00)$ & $49.00(20.25-59.50)$ & .303 \\
SOD-2 & $51.50(17.75-59.50)$ & $48.00(16.50-64.50)$ & .843 \\
EGFR & $\mathbf{1 9 . 0 0 ( 1 5 . 0 0 - 4 8 . 0 0 )}$ & $\mathbf{5 6 . 5 0 ( 4 1 . 2 5 - 6 6 . 0 0 )}$ & $\mathbf{. 0 1 9}$ \\
HER-2 & & & \\
\hline
\end{tabular}

the cancerous, as compared to unaffected tissue [21], the others investigated either whole SOD activity, irrespective of isoenzymes, or SOD proteins (by immunohistochemistry (IHC) or Western blot). Some criticism has been raised about the specificity of antibodies to SOD-2 [20]. Our results are consistent with the experimental observation that SOD activity may suppress the malignant phenotype [20].

The role of HO-1 in carcinogenesis has not yet been well elucidated [22]. Our data support the hypothesis that the expression of the enzyme is defective in lung cancer tissue and are in agreement with a recent study [23] showing reduced HO-1 expression (by IHC methods) in the alveolar macrophages of patients with NSCLC, as compared to controls.

Overexpression of EGFR and HER-2 receptors in NSCLC samples has been reported in many series, ranging from 43 to $89 \%$ and from 16 to $57 \%$ of tumors and patients, respectively (reviewed in [24]). Inconsistent results may reflect differences in analytical techniques, procedures, and study populations. Several methods are available to evaluate the tissue expression of these receptors, including those looking at proteins (IHC, Western blotting or enzyme immunoassay), RNA (Northern blot analyses and RT-PCR), or DNA (fluorescence in situ hybridization (FISH) or quantitative PCR) levels. The most commonly used technique was IHC, whose standardization is far to be achieved [25]. The Real-Time qRT-PCR we used for our analyses is an accurate technique that may allow the direct comparison of expression levels of target genes in both the cancerous and unaffected tissues. Our study, even demonstrating similar expression profiles of EGFR and HER-2 in cancerous and unaffected tissues, confirmed that EGFR is overexpressed in SCC, as compared to ADC samples, whereas HER-2 displays an opposite behaviour $[25,26]$. This may have therapeutic consequences, as both the proteins have been validated as clinically relevant targets, and several different types of agents inhibiting these receptors are currently under development [27].

In this study, the overall five-year survival rate $(56 \%$ considering only patients at stages I and II) was consistent with previous observation $[19,28]$. However, the HER2 expression ratio in the cancerous versus the unaffected tissue was a significant predictor of the 5-year prognosis. At the time of surgery, five-year survivors showed values of HER-2 transcript ratio that were on average about 30\% of the values observed in patients undergoing death. At the end of the follow-up period, subjects over-expressing the receptor in the cancerous tissue were deceased by $80 \%$, whereas those with lower expression ratios were still alive by $80 \%$. The Cox multivariate analysis allowed us to exclude any interference by tumor stage, histology, and age. A main limitation of this study was its small sample size. However, the extent of deviation from expected 5-year survival in both the patient groups ensures the statistical power of the results (alpha $=5 \%$, beta $=80 \%)$. Our data are in agreement with previous studies, mostly relying on IHC characterizations and giving a meta-analytical hazard ratio of $1.5(95 \%$ confidence interval 1.29-1.86) for worse prognosis associated to HER-2 overexpression [24]. The mechanisms linking HER-2 expression to a bad prognosis rely on a pleiotropic cascade of effects secondary to HER-2 activation. These include (i) increase of cell proliferation and survival and perturbation of differentiation, dependent on the activation of the phosphatidylinositol 3-kinase-AKT and the ras/mitogenactivated protein kinase pathways $[29,30]$, (ii) increased angiogenesis, as a consequence of the ability of HER-2 to modulate the equilibrium between proangiogenic (VEGF, IL-8, angiopoietin-2) and antiangiogenic factors [31].

\section{Conclusions}

Obtained results demonstrate that in biopsy samples from clinical early NSCLC, the expression levels of some antioxidant genes are depressed as compared to the surrounding healthy tissue. Interestingly, the patients' prognosis shows a significant inverse association to the ratio of HER-2 transcript levels in the cancerous versus the unaffected tissue.

However, owing to the small sample size, caution must be exercised in the interpretation of results that are to be 
considered as preliminary. Further confirmation by larger studies is needed, before observed findings may be translated into biomarkers useful to improve clinical managing of NSCLC cases.

\section{Acknowledgment}

This work was funded by a grant of the Italian Ministry of Health, code PMS/26/03.

\section{References}

[1] A. Jemal, R. Siegel, E. Ward, et al., "Cancer statistics," CA: Cancer Journal for Clinicians, vol. 58, no. 2, pp. 71-96, 2008.

[2] D. G. Beer, S. L. Kardia, C. C. Huang, et al., "Geneexpression profiles predict survival of patients with lung adenocarcinoma," Nature Medicine, vol. 8, no. 8, pp. 816-824, 2002.

[3] H. Y. Chen, S. L. Yu, C. H. Chen, et al., "A five-gene signature and clinical outcome in non-small-cell lung cancer," The New England Journal of Medicine, vol. 356, no. 1, pp. 11-20, 2007.

[4] A. Potti, S. Mukherjee, R. Petersen, et al., "A genomic strategy to refine prognosis in early-stage non-small-cell lung cancer," The New England Journal of Medicine, vol. 355, no. 6, pp. 570 $580,2006$.

[5] J. E. Klaunig and L. M. Kamendulis, "The role of oxidative stress in carcinogenesis," Annual Review of Pharmacology and Toxicology, vol. 44, pp. 239-267, 2004.

[6] R. Franco, O. Schoneveld, A. G. Georgakilas, and M. I. Panayiotidis, "Oxidative stress, DNA methylation and carcinogenesis," Cancer Letters, vol. 266, no. 1, pp. 6-11, 2008.

[7] M. Valko, C. J. Rhodes, J. Moncol, M. Izakovic, and M. Mazur, "Free radicals, metals and antioxidants in oxidative stressinduced cancer," Chemico-Biological Interactions, vol. 160, no. 1, pp. 1-40, 2006.

[8] L. Behrend, G. Henderson, and R. M. Zwacka, "Reactive oxygen species in oncogenic transformation," Biochemical Society Transactions, vol. 31, no. 6, pp. 1441-1444, 2003.

[9] L. E. Otterbein and A. M. K. Choi, "Heme oxygenase: colors of defense against cellular stress," American Journal of Physiology, vol. 279, no. 6, pp. L1029-L1037, 2000.

[10] L. E. Fredenburgh, M. A. Perrella, and S. A. Mitsialis, "The role of heme oxygenase-1 in pulmonary disease," American Journal of Respiratory Cell and Molecular Biology, vol. 36, no. 2, pp. 158-165, 2007.

[11] I. Fridovich, "Superoxide radical and superoxide dismutases," Annual Review of Biochemistry, vol. 64, pp. 97-112, 1995.

[12] E. Giannoni, F. Buricchi, G. Grimaldi, et al., "Redox regulation of anoikis: reactive oxygen species as essential mediators of cell survival," Cell Death and Differentiation, vol. 15, no. 5, pp. 867-878, 2008.

[13] H. L. Chan, H. C. Chou, M. Duran, et al., "Major role of EGFR and SRC kinases in promoting oxidative stress-dependent loss of adhesion and apoptosis in epithelial cells," The Journal of Biological Chemistry, vol. 285, no. 7, pp. 4307-4318, 2010.

[14] K. Nakai, K. Yoneda, J. Igarashi, T. Moriue, H. Kosaka, and Y. Kubota, "Angiotensin II enhances EGF receptor expression levels via ROS formation in HaCaT cells," Journal of Dermatological Science, vol. 51, no. 3, pp. 181-189, 2008.

[15] S. V. Sharma and J. Settleman, "ErbBs in lung cancer," Experimental Cell Research, vol. 315, no. 4, pp. 557-571, 2009.
[16] S. R. Hubbard and W. T. Miller, "Receptor tyrosine kinases: mechanisms of activation and signaling," Current Opinion in Cell Biology, vol. 19, no. 2, pp. 117-123, 2007.

[17] Y. Yarden and M. X. Sliwkowski, "Untangling the ErbB signaling network," Nature Reviews Molecular Cell Biology, vol. 2, no. 2, pp. 127-137, 2001.

[18] J. Vandesompele, K. De Preter, F. Pattyn, et al., "Accurate normalization of real-time quantitative RT-PCR data by geometric averaging of multiple internal control genes," Genome Biology, vol. 3, no. 7, Article ID RESEARCH0034, 2002.

[19] C. F. Mountain, "Revisions in the international system for staging lung cancer," Chest, vol. 111, no. 6, pp. 1710-1717, 1997.

[20] V. L. Kinnula and J. D. Crapo, "Superoxide dismutases in malignant cells and human tumours," Free Radical Biology and Medicine, vol. 36, no. 6, pp. 718-744, 2004.

[21] J. Chung-man Ho, S. Zheng, S. A. Comhair, C. Farver, and S. C. Erzurum, "Differential expression of manganese superoxide dismutase and catalase in lung cancer," Cancer Research, vol. 61, no. 23, pp. 8578-8585, 2001.

[22] A. Jozkowicz, H. Was, and J. Dulak, "Heme oxygenase-1 in tumors: is it a false friend?" Antioxidants 'Redox Signaling, vol. 9, no. 12, pp. 2099-2117, 2007.

[23] P. Boschetto, E. Zeni, L. Mazzetti, et al., "Decreased hemeoxygenase (HO)-1 in the macrophages of non-small cell lung cancer," Lung Cancer, vol. 59, no. 2, pp. 192-197, 2008.

[24] A. -P. Meert, B. Martin, M. Paesmans, et al., "The role of HER2/neu expression on the survival of patients with lung cancer: a systematic review of the literature," British Journal of Cancer, vol. 89, no. 6, pp. 959-965, 2003.

[25] A. Onn, A. M. Correa, M. Gilcrease, et al., "Synchronous overexpression of epidermal growth factor receptor and HER2-neu protein is a predictor of poor outcome in patients with stage $i$ non-small cell lung cancer," Clinical Cancer Research, vol. 10, no. 1 I, pp. 136-143, 2004.

[26] F. R. Hirsch, G. V. Scagliotti, C. J. Langer, M. Varella-Garcia, and W. A. Franklin, "Epidermal growth factor family of receptors in preneoplasia and lung cancer: perspectives for targeted therapies," Lung Cancer, vol. 41, supplement 1, pp. S29-S42, 2003.

[27] P. S. Sharma, R. Sharma, and T. Tyagi, "Receptor tryosine kinase inhibitors as potent weapons in war against cancers," Current Pharmaceutical Design, vol. 15, no. 7, pp. 758-776, 2009.

[28] J. Pfannschmidt, T. Muley, H. Bulzebruck, H. Hoffmann, and H. Dienemann, "Prognostic assessment after surgical resection for non-small cell lung cancer: experiences in 2083 patients," Lung Cancer, vol. 55, no. 3, pp. 371-377, 2007.

[29] J. Baselga, J. Albanell, M. A. Molina, and J. Arribas, "Mechanism of action of trastuzumab and scientific update," Seminars in Oncology, vol. 28, no. 5, supplement 16, pp. 4-11, 2001.

[30] J. Schlessinger, "Common and distinct elements in cellular signaling via EGF and FGF receptor," Science, vol. 306, no. 5701, pp. 1506-1507, 2004.

[31] X. F. Wen, G. Yang, W. Mao, et al., "HER2 signaling modulates the equilibrium between pro- and antiangiogenic factors via distinct pathways: implications for HER2-targeted antibody therapy," Oncogene, vol. 25, no. 52, pp. 6986-6996, 2006. 

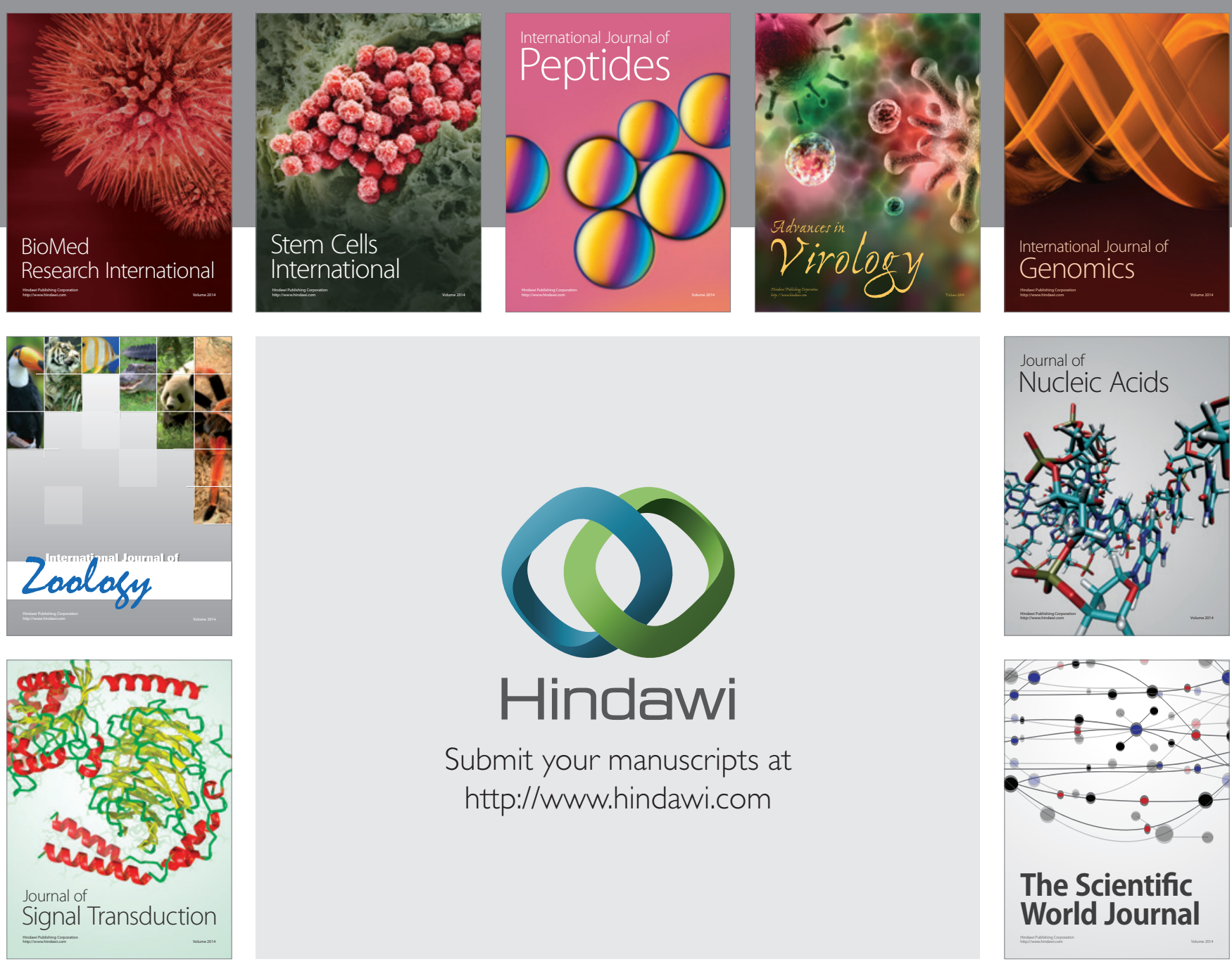

Submit your manuscripts at

http://www.hindawi.com
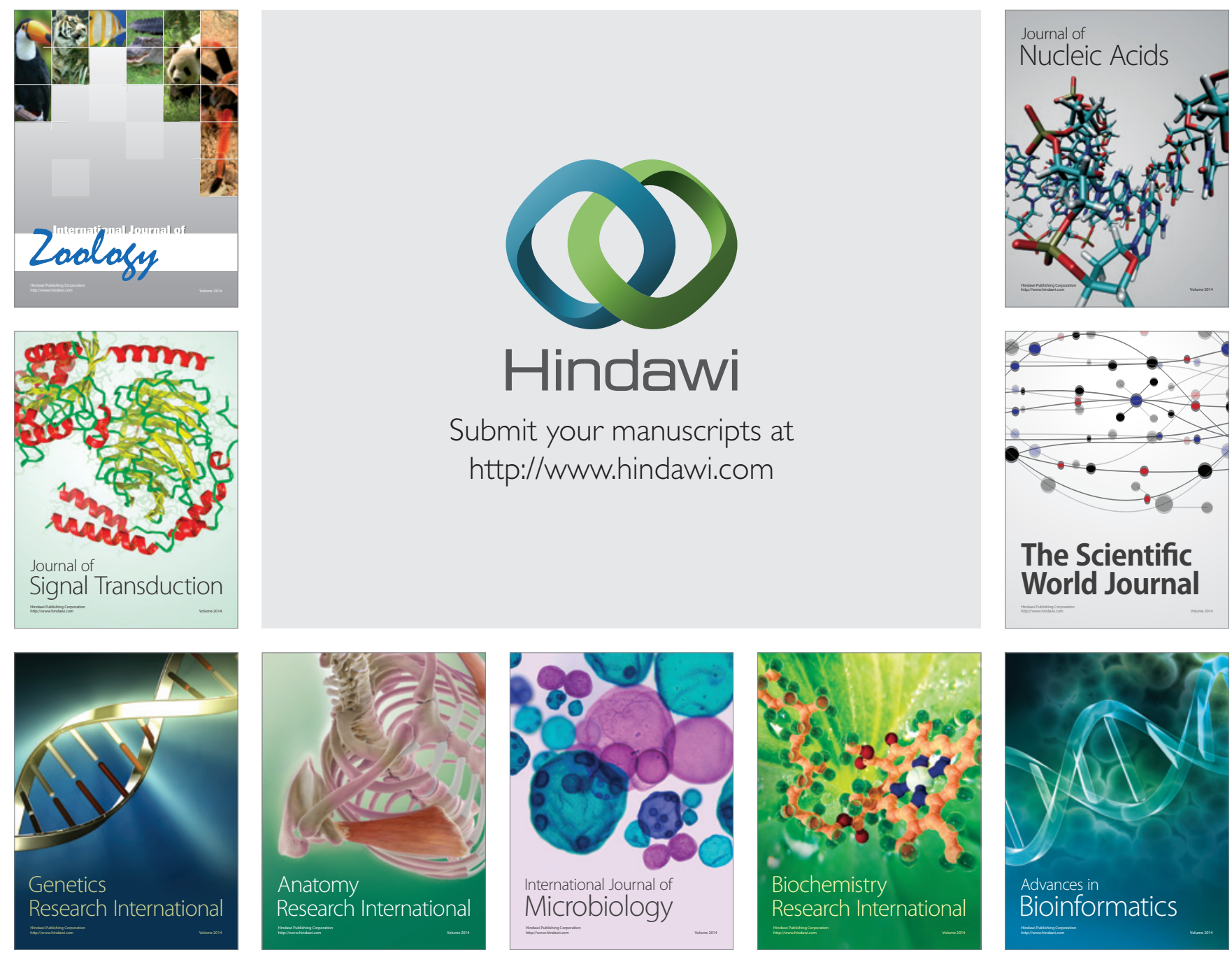

The Scientific World Journal
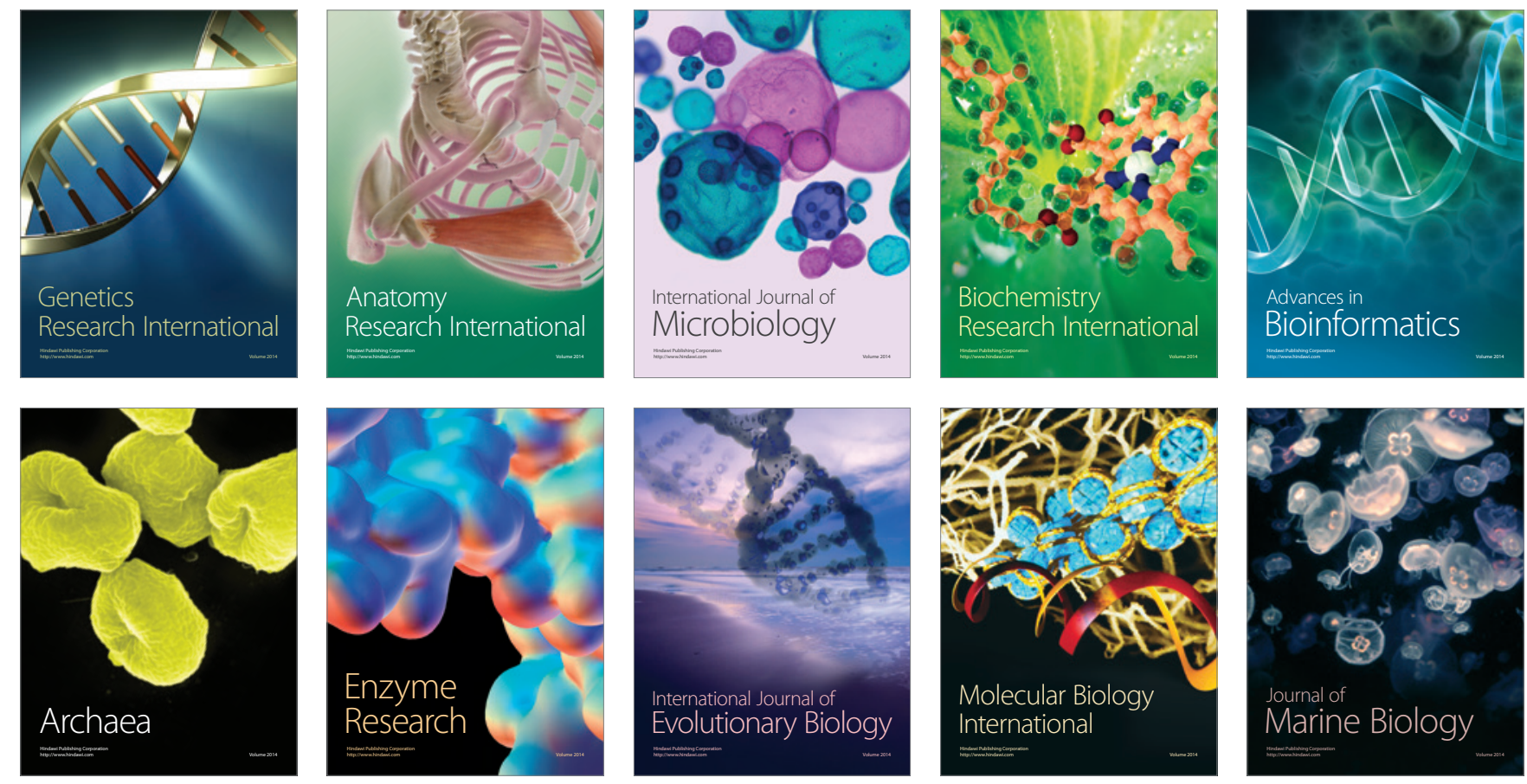